Ophthalmologe 2022 $\cdot 119: 410-413$ https://doi.org/10.1007/s00347-021-01489-z Eingegangen: 6. Juli 2021

Angenommen: 9. August 2021

Online publiziert: 21. September 2021

(c) Der/die Autor(en) 2021

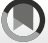

Check tor
updates

\section{Geteilter Tumor des Ober- und Unterlides}

\author{
P. J. Gaca' $\cdot$ A. Doulis' $\cdot$ P. A. Wawer Matos ${ }^{1,2} \cdot$ M. Lewandowicz ${ }^{3}$ A. C. Rokohl ${ }^{1,2}$. \\ L. M. Heindl ${ }^{1,2}$ \\ 'Zentrum für Augenheilkunde, Medizinische Fakultät und Universitätsklinikum Köln, Universität zu Köln, \\ Köln, Deutschland \\ ${ }^{2}$ Centrum für Integrierte Onkologie (CIO) Aachen-Bonn-Köln-Düsseldorf, Köln, Deutschland \\ ${ }^{3}$ Abteilung für onkologische Chirurgie, Multidisziplinäres M. Copernicus Woiwodschaftszentrum für \\ Onkologie und Traumatologie in Lodz, Lodz, Polen
}

\section{Anamnese}

Eine 12-jährige Patientin stellte sich in unserer ophthalmoonkologischen Sprechstunde mit einer seit Geburt vorhandenen, pigmentierten und seit 1 Jahr in der Dicke progredienten, schmerzlosen Raumforderung am linken medialen Ober- und Unterlid vor. Die Augenanamnese war bis auf einen geringen myopen Astigmatismus leer. Anamnestisch bestanden keine Allgemeinerkrankungen, kein Trauma, keine B-Symptomatik oder andere Beschwerden. Bis auf positive Allergieanamnese gegen Pollen war die Patientin komplett gesund. Die Patientin nahm keinerlei Medikamente ein. Auch die Familienanamnese bezüglich ophthalmologischer Erkrankungen war leer.

\section{Klinischer Befund}

Die 12-jährige Patientin befand sich in einem sehr guten, altersüblichen Allgemeinund Ernährungszustand. Der Fernvisus lag beidseits mit bestmöglicher Refraktion bei 1,0. Die Motilität beider Augen war frei. In der weiteren klinischen ophthalmologischen Untersuchung zeigte sich am linken Auge eine schmerzlose, nicht druckdolente, scharf und regelmäßig begrenzte hellbraun pigmentierte, plakoide Raumforderung mit gering papillomatöser Oberfläche des medialen Ober- und Unterlides (- Abb. 1). Der Tumor imponierte durch die Lidspalte gleichsam geteilt. Der restliche vordere Augenabschnitt war unauffällig. Die Untersuchung des Augenfundus zeigte keinen pathologischen Befund.

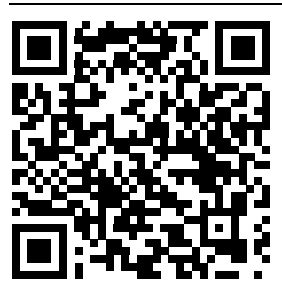

QR-Code scannen \& Beitrag online lesen

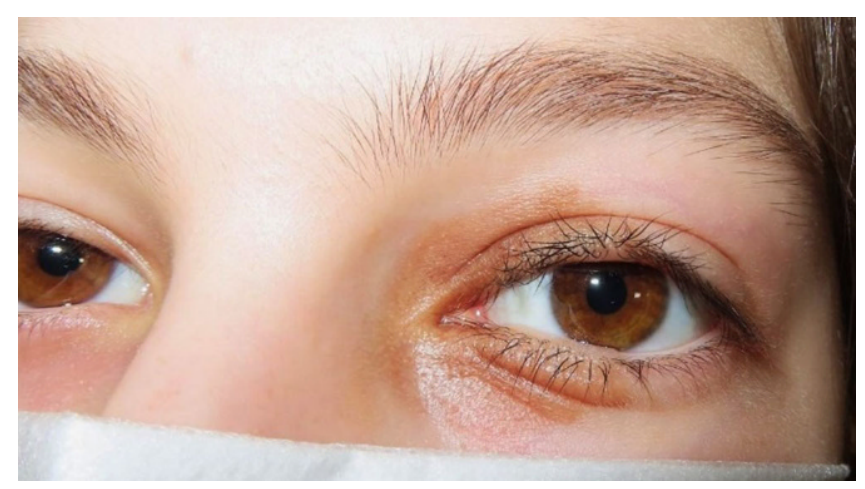

Abb. 1 <12-jährige Patientin mit hellbraun pigmentierter Raumforderung des medialen Oberund Unterlides 
Die Diagnose lautet kongenitaler geteilter melanozytärer Nävus des Ober- und Unterlides - auch als "kissing nevus", "split ocular nevus" und "panda nevus" bezeichnet.

\section{Diskussion}

Geteilte Nävi des Ober- und Unterlides sind seltene melanozytäre Veränderungen der Augenlider. Sie wurden erstmals 1919 von Fuchs beschrieben [3]. In der Literatur wurden bisher etwa 120 solcher Fälle beschrieben [5]. Bei einem geteilten Nävus sind am oberen und unteren Lid gleichzeitig 2 getrennte Nävi vorhanden, die bei geschlossenem Auge eine Einheit bilden (-Abb. 2). Geteilte Nävi sind in der Regel im medialen Bereich des Augenlides lokalisiert, obwohl sie auch in den medialen Lidwinkeln auftreten können. Die Augenlider sind bei Weitem die häufigste Lokalisation eines geteilten Nävus. Geteilte Nävi wurden auch an anderen Stellen des Körpers beschrieben. Der Penis und die Fingerzwischenräume sind weitere jedoch viel seltenere Lokalisationen gepaarter Läsionen [5].

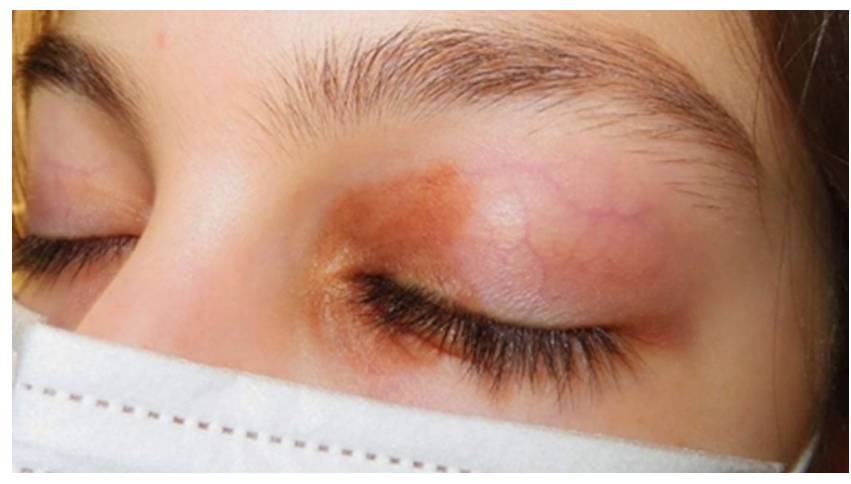

Abb. $2 \triangleleft$ Bei einem geteilten Nävus sind am oberen und unteren Lid gleichzeitig 2 getrennte Nävi vorhanden, die bei geschlossenem Auge eine Einheit bilden

Die Manifestation an gegenüberliegenden Arealen von Ober- und Unterlid ist Ausdruck der Embryonalentwicklung. Die Augenlider fusionieren in der 9. bis 10 . Embryonalwoche (Woche post conceptionem), (38-40 mm, Scheitel-Steiß-Länge). Die Anlage des Nävus erfolgt im Stadium der fusionierten Lider [1, 4].

Als Abkömmlinge des Neuroektoderms wandern Melanozytenvorläufer während der Embryogenese als Melanoblasten aus der dorsalen Neuralleiste über die Dermis in die Epidermis in die verschiedenen Regionen des Embryos ein [1, 4]. Zwischen der 12. und 14. Embryonalwoche $(100-120 \mathrm{~mm})$ erfolgt die Wanderung zur Unterseite der embryonalen Epidermis der Kopfhaut und des Gesichts [4]. Aufgrund ihres Ursprungs in der Neuralleiste können Melanoblasten lokalisierte Proliferationen in den intradermalen, junktionalen und Compoundnävi zeigen [4].

Während der 20. Embryonalwoche beginnen sich die Augenlider zu trennen, nachdem sich Lipide in der dermoepidermalen Junktionszone ansammeln. Durch die spätere Trennung von Ober- und Un-
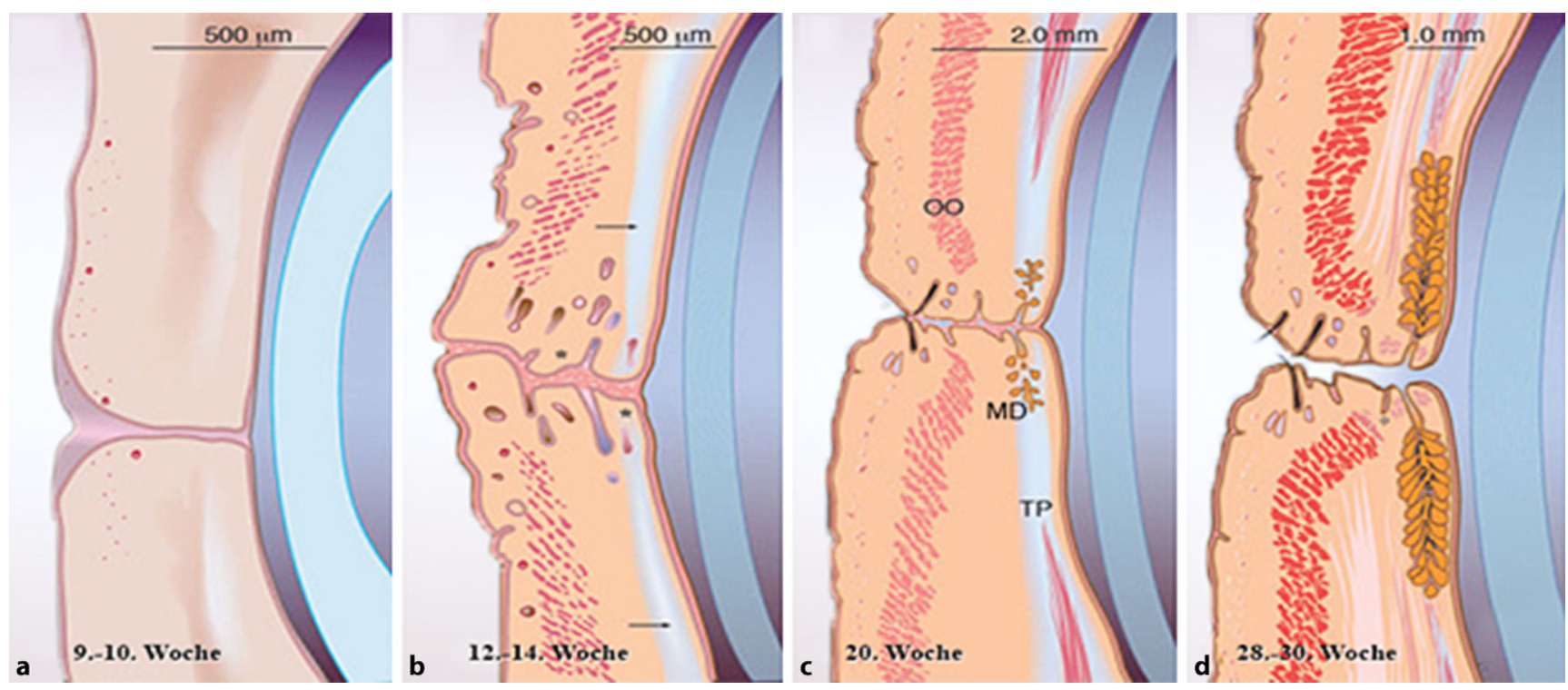

Abb. $3 \Delta$ Embryonalentwicklung des Augenlides. a 9.-10. Embryonalwoche (38-40 mm). Die Augenlider fusionieren. Mesenchymale Zellen kondensieren und formen Primordien der Wimpernfollikel und ihrer Anhängsel, der Tarsalplatte (TP) und des Orbicularis oculi $(O O)$. b 12. bis 14 . Embryonalwoche $(100-120 \mathrm{~mm})$. Das Augenlid ist in separate Schichten unterteilt. Am Lidrand sind rudimentäre Wimpern, Talg- und Schweißdrüsen erkennbar sowie eine primordiale Tarsalplatte (Pfeil). c 20. Embryonalwoche.Obwohl die Augenlidervon außen noch verwachsen zu sein scheinen, hat die Trennung bereits begonnen. Eine Verzweigung der Meibom-Drüsen, Verlängerung der Tarsalplatte, Weiterentwicklung von Orbicularis oculi und Wimpernfollikel findet statt. d 28. bis 30. Embryonalwoche (180-200 mm). Die Lider sind inzwischen vollständig getrennt. Meibom-Drüsen nehmen an Länge zu und sind in zwei Drittel der Länge der Tarsalplatte vorhanden. (Mod. nach Tawfik et al. [7]) 
terlid in der 28. bis 30. Embryonalwoche (180-200 mm) kommt es zur Teilung des Nävus [1]. Daraus kann man ableiten, dass Nävi in dieser Lokalisation spätestens zu diesem Zeitpunkt vorhanden sein müssen (- Abb. 3). Interessanterweise wurde kein histologischer Unterschied in nävalen Subtypen (junktional, intradermal, compound) zwischen Ober- und Unterlid beschrieben [2].

Die Differenzialdiagnose des geteilten Nävus umfasst Papillome, Neurofibrome, Epitheliome. In der Vergangenheit waren es Tuberkulome und Syphilide [2].

\section{I) Diagnose: Kongenitaler geteilter melanozytärer Nävus}

Basierend auf dem Durchmesser (in cm), können die Nävi wie folgt eingeteilt werden:

a) klein $(<1,5 \mathrm{~cm})$, b) mittel $(1,5-$ $19,9 \mathrm{~cm}), c)$ groß $(>20 \mathrm{~cm})$.

Eine maligne Entartung bei kleinen oder mittleren Nävi wurde bisher nicht beobachtet [2]. Das Risiko einer malignen Transformation in ein malignes Melanom ist bei großen kongenitalen Nävi gut bekannt, bei kleinen und mittelgroßen Läsionen, die häufiger bei geteilten Nävi auftreten, ist es jedoch weniger klar [6]. Die tatsächliche Inzidenz der malignen Entartung in der Literatur ist sehr variabel und reicht von 2-40\% je nach Dauer der Nachbeobachtung mit einem Durchschnitt von $14 \%$ für das gesamte Leben [6].

Kissing-Nävi der Augenlider müssen regelmäßig kontrolliert werden. Eine therapeutische Entfernung (Laser-, Kryo, chirurgische Exzision mit ophthalmoplastischer Deckung) ist aufgrund des niedrigen Entartungsrisikos selten medizinisch indiziert. Sie kann bei sekundärer Amblyopie bei Ptosis, Epiphora oder Kompression der Puncta lacrimalia oder bei kosmetischem Wunsch erfolgen.

\section{Fazit für die Praxis}

Zusammenfassend sind geteilte Nävi des Ober- und Unterlides seltene melanozytäre Veränderungen der Augenlider, die regelmäßig kontrolliert werden müssen. Eine therapeutische Entfernung (Laser-, Kryo, chirurgische Exzision mit ophthalmoplastischer Deckung) ist aufgrund des niedrigen Ent- artungsrisikos selten medizinisch indiziert. Sie kann bei sekundärer Amblyopie bei Ptosis, Epiphora oder Kompression der Puncta lacrimalia oder bei kosmetischem Wunsch erfolgen.

\section{Korrespondenzadresse}

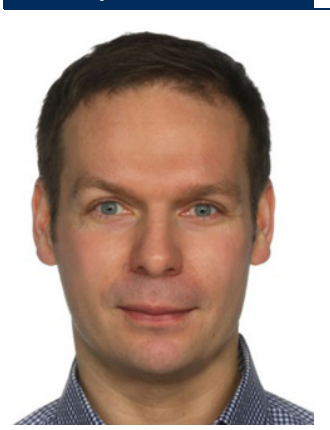

\section{Dr. med. P. J. Gaca}

Zentrum für Augenheilkunde, Medizinische Fakultät und Universitätsklinikum Köln, Universität zu Köln Kerpener Str. 62, 50937 Köln, Deutschland piotrjakub.gaca@gmail.com

Funding. Open Access funding enabled and organized by Projekt DEAL.

\section{Einhaltung ethischer Richtlinien}

Interessenkonflikt. P.J. Gaca, A. Doulis, P.A. Wawer Matos, M. Lewandowicz, A.C. Rokohl und L.M. Heindl geben an, dass kein Interessenkonflikt besteht.

Für diesen Beitrag wurden von den Autoren keine Studien an Menschen oder Tieren durchgeführt. Für Bildmaterial oder anderweitige Angaben innerhalb des Manuskripts, über die Patienten zu identifizieren sind, liegt von ihnen eine schriftliche Einwilligung vor.

Open Access. Dieser Artikel wird unter der Creative Commons Namensnennung 4.0 International Lizenz veröffentlicht, welche die Nutzung, Vervielfältigung, Bearbeitung, Verbreitung und Wiedergabe in jeglichem Medium und Format erlaubt, sofern Sie den/die ursprünglichen Autor(en) und die Quelle ordnungsgemäß nennen, einen Link zur Creative Commons Lizenz beifügen und angeben, ob Änderungen vorgenommen wurden.

Die in diesem Artikel enthaltenen Bilder und sonstiges Drittmaterial unterliegen ebenfalls der genannten Creative Commons Lizenz, sofern sich aus der Abbildungslegende nichts anderes ergibt. Sofern das betreffende Material nicht unter der genannten Creative Commons Lizenz steht und die betreffende Handlung nicht nach gesetzlichen Vorschriften erlaubt ist, ist für die oben aufgeführten Weiterverwendungen des Materials die Einwilligung des jeweiligen Rechteinhabers einzuholen.
Weitere Details zur Lizenz entnehmen Sie bitte der Lizenzinformation auf http://creativecommons.org/ licenses/by/4.0/deed.de.

\section{Literatur}

1. Andersen H, Ehlers N, Matthiessen ME (1965) Histochemistry and development of the human eyelids. Acta Ophthalmol 43:642-668

2. Ehlers N (1969) Divided nevus. Acta Ophthalmol 47:1004-1011

3. Fuchs A (1960) Divided nevi of the skin of the eyelid. Klin Monbl Augenheilkd Augenarztl Fortbild 137:504-505

4. Hamming N (1983) Anatomy and embryology of the eyelids: a review with special reference to the development of divided nevi. Pediatr Dermatol 1:51-58

5. Jia R, Zhu H, Lin M et al (2012) Clinicopathological characteristics and surgical outcomes of divided nevus of the eyelids: a decade's experience on 73 cases. Ann Plast Surg 68:166-170

6. Mcdonnell PJ, Mayou BJ (1988) Congenital divided naevus of the eyelids. Br J Ophthalmol 72:198-201

7. Tawfik HA, Abdulhafez MH, Fouad YA, Dutton JJ (2016) Embryologic and Fetal Development of the Human Eyelid. Ophthal Plast Reconstr Surg 32(6):407-414 
Hier steht eine Anzeige.

黑 Springer 